\title{
Decreased osteoclastogenesis, osteoblastogenesis and low bone mass in a mouse model of type 2 diabetes
}

\author{
FEI XU, YONGHUI DONG, XIN HUANG, MI LI, LIANG QIN, YE REN, \\ FENGJING GUO, ANMIN CHEN and SHILONG HUANG \\ Department of Orthopedics, Tongji Hospital, Tongji Medical College, \\ Huazhong University of Science and Technology, Wuhan, Hubei 430000, P.R. China
}

Received September 21, 2013; Accepted October 25, 2013

DOI: $10.3892 / \mathrm{mmr} .2014 .2430$

\begin{abstract}
The effect of type 2 diabetes mellitus (T2DM) on bone is controversial. Therefore, the present study investigated whether T2DM causes osteoporosis and explored the underlying mechanisms involved in this process. The effects of T2DM on bone physiology were analyzed in a mouse model of T2DM; KK/Upj-Ay/J (KK-Ay) mice develop diabetes after 8 weeks and exhibit stable diabetes symptoms and signs after 10 weeks when fed a KK-Ay mouse maintenance fodder. Diabetic mice exhibited hyperglycemia, hyperinsulinemia and increased body and fat pad weight in comparison with C57BL/6 non-diabetic mice. Furthermore, diabetic mice demonstrated low bone weight and bone mineral density in the femur, tibia and fifth lumbar vertebra. Using von Kossa and tartrate-resistant acid phosphatase (TRAP) staining, alkaline phosphatase and TRAP activity analyses and gene profiling it was demonstrated that osteoblastogenesis and osteoclastogenesis were impaired in diabetic mice. To evaluate the bone biomechanics, the ultimate load of the bone was analyzed. It was found that the ultimate load of the tibia in diabetic mice was lower than that in the controls. The results from the present study suggest that bone metabolism is impaired in T2DM, resulting in decreased osteoblastogenesis, osteoclastogenesis and bone mass.
\end{abstract}

\section{Introduction}

The number of patients with type 2 diabetes mellitus (T2DM) increases annually in China, as well as in other countries. Patients with T2DM have hyperinsulinemia, due to impaired cellular sensitivity to insulin, and hyperglycemia, due to

Correspondence to: Professor Shilong Huang, Department of Orthopedics, Tongji Hospital, Tongji Medical College, Huazhong University of Science and Technology, 1095 Jiefang Avenue, Wuhan, Hubei 430000, P.R. China

E-mail: doctorhsl@gmail.com

Key words: osteoblastogenesis, osteoclastogenesis, osteoporosis, type 2 diabetes mellitus insulin resistance. Hyperglycemia and hyperinsulinemia cause numerous complications, including nephropathy, neuropathy and retinopathy. Osteoporosis, along with an elevated risk of fragility fractures, has been observed in patients with T2DM (1). Epidemiological evidence has also demonstrated that there is an increased risk of hip, humerus and foot fractures in diabetic subjects (2). Furthermore, patients with T2DM have a higher risk of nonunion fracture, which leads to burdens on society (3).

Impaired bone turnover as a result of diabetes is widely accepted (4); however, there are limited data on the underlying mechanism. A number of studies have reported that type 1 diabetes changes bone remodeling by impairing bone formation, leading to osteopenia $(2,5)$. By contrast, bone loss in T2DM is less accepted, with reports of bone mineral density (BMD) ranging from low to high in T2DM (6-8). Therefore, the present study investigated whether T2DM causes osteoporosis and explored the underlying mechanisms involved in this process by performing histological, cellular and biomechanical experiments on male $\mathrm{KK} / \mathrm{Upj}-\mathrm{Ay} / \mathrm{J}$ (KK-Ay) mice.

\section{Materials and methods}

Animals. Male KK-Ay mice with a C57BL/6 background and male C57BL/6 mice were purchased from Beijing HFK Bio-Technology, Co., Ltd. (Beijing, China), aged 5-6 weeks. Male KK-Ay mice develop T2DM between 7 and 8 weeks of age. The C57BL/6 mice were non-diabetic and served as controls. Animals were housed with a 12-h light/dark cycle and fed KK-Ay Diet 1K65 (HFK Bio-Technology, Co., Ltd.) and water ad libitum. All procedures were approved by the Animal Care and Use Committee, Tongji Medical College, Huazhong University of Science and Technology (Wuhan, China).

At the age of 7-8 weeks, nonfasting blood glucose measurements were obtained using blood from the lateral saphenous vein and a glucometer (Bayer, Whippany, NJ, USA). Mice with blood glucose levels $>300 \mathrm{mg} / \mathrm{dl}$ were considered diabetic. Animals were euthanized by $\mathrm{CO}_{2}$ inhalation and cervical dislocation 4 weeks subsequent to confirmation of diabetes. Weights of the bilateral inguinal fat and right tibiae were recorded. Bone marrow was harvested 
Table I. Primer sequences.

\begin{tabular}{|c|c|c|c|}
\hline Gene & Primer & Product size (bp) & Accession no. \\
\hline $18 \mathrm{~S}$ & $\begin{array}{l}\text { F: TTCGAACGTCTGCCCTATCAA } \\
\text { R: ATGGTAGGCACGGGGACTA }\end{array}$ & 50 & M35283.1 \\
\hline RUNX2 & $\begin{array}{l}\text { F: GACTGTGGTTACCGTCATGGC } \\
\text { R: ACTTGGTTTTTCATAACAGCGGA }\end{array}$ & 84 & NM_001146038 \\
\hline ALP & $\begin{array}{l}\text { F: GCCTTACCAACTCTTTTGTGCC } \\
\text { R: GCTTGCTGTCGCCAGTAAC }\end{array}$ & 61 & NM_007431 \\
\hline $\mathrm{OCN}$ & $\begin{array}{l}\text { F: CTGACCTCACAGATCCCAAGC } \\
\text { R: TGGTCTGATAGCTCGTCACAAG }\end{array}$ & 187 & NM_031368 \\
\hline Cathepsin k & $\begin{array}{l}\text { F: GAAGAAGACTCACCAGAAGCAG } \\
\text { R: CTGTATTCCCCGTTGTGTAGC }\end{array}$ & 136 & NM_007802 \\
\hline TRAP & $\begin{array}{l}\text { F: CACTCCCACCCTGAGATTTGT } \\
\text { R: CATCGTCTGCACGGTTCTG }\end{array}$ & 118 & NM_001102405 \\
\hline
\end{tabular}

RUNX2, runt-related transcription factor 2; ALP, alkaline phosphatase; OCN, osteocalcin; TRAP, tartrate-resistant acid phosphatase; F, forward; R, reverse.

from both femora. Left tibiae and L5 vertebrae were analyzed using micro-computed tomography (mCT).

Serum analysis. Blood serum samples were obtained from blood collected by cardiac puncture immediately subsequent to euthanasia. Serum measurements of glucose, insulin, triglyceride (TG), cholesterol (TC), alkaline phosphatase (ALP), tartrate-resistant acid phosphatase (TRAP) and osteocalcin (OCN) were performed using a glucometer and ELISA kits (Wuhan Boster Biological Technology, Ltd., Wuhan, China).

mCT analysis. mCT analysis was performed using a Scanco $\mu \mathrm{CT} 50$ (Scanco Medical AG, Bassersdorf, Switzerland). Scans were performed under the following conditions: Voltage, $70 \mathrm{KVp}$; current, $110 \mu \mathrm{A}$; increment, $5 \mu \mathrm{m}$; threshold value, 289. Parameters, including images and BMD, describing the tibiae and vertebrae were computed using the Scanco $\mu$ CT 50 system.

Osteoblastogenesis assay. Bone marrow stromal cells were collected and seeded at a density of $2.5 \times 10^{5} / \mathrm{cm}^{2}$ in 24 -well plates with osteoblast differentiation-inducing media containing $\alpha$-Minimum Essential Media ( $\alpha$-MEM; Gibco-BRL, Carlsbad, CA, USA) supplemented with $15 \%$ fetal bovine serum (FBS; Gibco-BRL), $100 \mu \mathrm{M}$ ascorbate phosphate, $5 \mathrm{M} \beta$-glycerol phosphate and $10 \mathrm{nM}$ dexamethasone. The media were changed every 2 days. Cells were harvested after 7, 14 and 21 days for von Kossa staining and measurement of ALP activity.

Osteoclastogenesis assay. Primary bone marrow cells were collected and seeded at a density of $2.5 \times 10^{5} / \mathrm{cm}^{2}$ in the presence of $\alpha$-MEM (Gibco-BRL) supplemented with $15 \%$ FBS (Gibco-BRL). Floating, non-adherent cells were harvested after $24 \mathrm{~h}$ and seeded at a density of $2 \times 10^{5} / \mathrm{cm}^{2}$ into 48 -well plates using medium supplemented with $50 \mathrm{ng} / \mathrm{ml}$ macrophage colony-stimulating factor and $50 \mathrm{ng} / \mathrm{ml}$ receptor activator of nuclear factor $\kappa$-B ligand (RANKL; R\&D
Systems, Minneapolis, MN, USA). The media were changed every 2 days. After 4 days of growth, cells were harvested for TRAP staining and measurement of TRAP activity.

ALP and TRAP activity measurement. To measure ALP activity, the harvested cells were washed with phosphate-buffered saline twice, prior to the addition of lysis buffer $[1.5 \mathrm{M}$ Tris- $\mathrm{HCl}$ (pH 9.2), 0.1 $\mathrm{M} \mathrm{ZnCl}_{2}, 0.5 \mathrm{M} \mathrm{MgCl}_{2}-6 \mathrm{H}_{2} \mathrm{O}$ and Triton $\mathrm{X}-100]$. The cells in each well were sonicated for $10 \mathrm{sec}$, and the sonicated samples were then added to substrate solution containing 4-nitrophenyl phosphate (Sigma, St Louis, MO, USA), $1.5 \mathrm{M}$ alkaline buffer solution (Sigma) and $\mathrm{H}_{2} \mathrm{O}$ and incubated for $20 \mathrm{~min}$ at $37^{\circ} \mathrm{C}$. The reaction was subsequently, terminated by the addition of $2 \mathrm{~N} \mathrm{NaOH}$. The absorbance of the samples was measured at $405 \mathrm{~nm}$ using a microplate reader (Multiskan ${ }^{\mathrm{TM}}$ FC Microplate Photometer, Thermo Fisher Scientific, Inc., Waltham, MA, USA).

For the measurement of TRAP activity, the harvested cells were fixed with $10 \%$ formalin for $10 \mathrm{~min}$ and $95 \%$ ethanol for $1 \mathrm{~min}$. A total of $100 \mu \mathrm{l}$ citrate buffer $(50 \mathrm{mM}$, $\mathrm{pH}$ 4.6) containing $10 \mathrm{mM}$ sodium tartrate and $5 \mathrm{mM}$ p-nitrophenylphosphate (Sigma) was subsequently added to the wells containing fixed cells in the plates. Following incubation with the citrate buffer for $1 \mathrm{~h}$, enzyme reaction mixtures in the wells were transferred to new plates containing an equal volume of $0.1 \mathrm{~N} \mathrm{NaOH}$. The absorbance was measured at $405 \mathrm{~nm}$ using a microplate reader (Multiskan FC Microplate Photometer, Thermo Fisher Scientific, Inc.). Each experiment was performed in triplicate.

RNA isolation and quantitative polymerase chain reaction (qPCR) analysis. Total RNA was isolated using TRIzol ${ }^{\circledR}$ Reagent (Invitrogen Life Technologies, Carlsbad, CA, USA). Gene expression analysis was performed using the Real-Time PCR Detection System IQ ${ }^{\text {TM }} 5$ (Bio-Rad, Hercules, CA, USA) and normalized against 18S RNA. Table I shows the primers used for qPCR. 
A

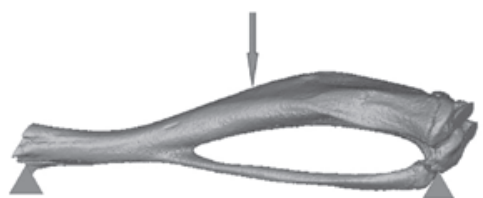

B

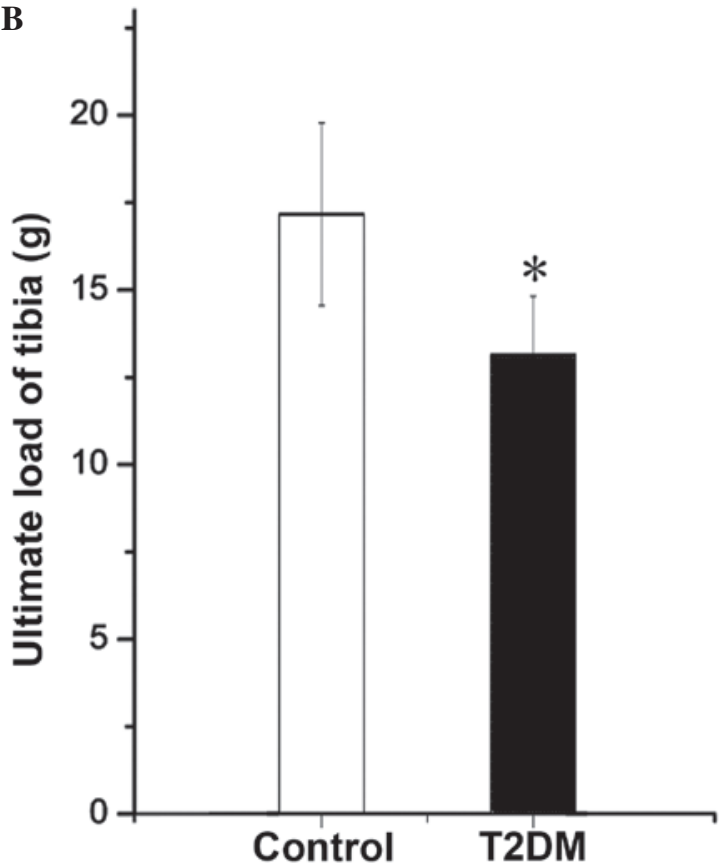

Figure 1. Biomechanical analysis. (A) Load was applied on the bone. (B) The ultimate load of the tibia in the T2DM mice was lower compared with that in the control mice. ${ }^{*} \mathrm{P}<0.05$, vs. control. T2DM, type 2 diabetes mellitus.

Biomechanical analysis. The strength of the tibial midshaft was measured using a universal testing machine (Instron, Norwood, MA, USA). Load was applied on the bone as shown in Fig. 1A, and the ultimate load for each sample was measured.

Statistical analysis. Statistical analyses were performed using the Student's t-test with all values expressed as the mean \pm standard deviation. All experiments were repeated in triplicate, and $\mathrm{P}<0.05$ was considered to indicate a statistically significant difference.

\section{Results}

KK-Ay mice are hyperglycemic, hyperinsulinemic, obese and exhibit high food consumption. KK-Ay mice exhibited elevated body weights with increased topical fat weight and high intake of food. They also had higher levels of serum insulin and non-fasting levels of glucose compared with the control mice (Fig. 2A). With regard to fat metabolism, KK-Ay mice had higher levels of serum TC and TG compared with the control animals, and the weight of the bilateral inguinal fat tissue from the KK-Ay mice was heavier than that from the control mice (Fig. 2B).

Alterations in serum bone turnover markers, including bone-specific alkaline phosphatase (BALP) enzyme activity, serum TRAP levels and serum OCN levels. In addition to higher serum glucose and insulin levels in diabetic mice, alterations were observed in the levels of certain bone formation markers. BALP enzyme activity was lower in diabetic mice than that in non-diabetic mice. Serum TRAP levels, a bone resorption marker, were unchanged in diabetic mice; however, the serum OCN levels were markedly lower in diabetic mice (Fig. 2C).

KK-Ay mice have lower BMD and trabecular and cortical bone mass. The femora and tibiae in the T2DM group were smaller and weighed less than those in the control group (Fig. 3). The bone mineralization density distribution of trabecular and cortical bone was similar in the KK-Ay and control mice; however, the density distribution in KK-Ay mice was slightly lower than that in the control mice (Fig. 3), and the T2DM group had a decreased bone content in the tibia compared with the control group. Data regarding cortical bone geometry from the midshaft of the tibia showed that T2DM bone also had a smaller cortical perimeter due to decreased cortical area and thickness (Fig. 3 and Table II).

Impaired osteoblastic and osteoclastic function in diabetic mice. To investigate the cellular mechanism for the low bone mass in T2DM mice, osteoblast and osteoclast biology was analyzed. The ALP activity of osteoblasts was reduced in T2DM mice (Fig. 4A). The results from the von Kossa staining showed impaired osteoblastogenesis in T2DM mice compared with control mice (Fig. 4B). Furthermore, runt-related transcription factor 2 (RUNX2), ALP and OCN levels were found to be reduced after 21 days of osteogenic differentiation (Fig. 4C). Osteoclast function was impaired in T2DM mice; the number of TRAP ${ }^{+}$and multinucleated osteoclasts and the TRAP activity of osteoclasts were reduced in T2DM mice (Fig. 5). In addition, the mRNA expressed by osteoclasts, including TRAP and cathepsin K (CTK), were reduced (Fig. 5).

Ultimate load of tibia is lower in T2DM mice. Biomechanical analysis demonstrated that the ultimate load of the tibia in the T2DM mice was lower than that in control mice (Fig. 1B).

\section{Discussion}

T2DM is know to affect bone remodeling, including decreased bone formation and impaired bone resorption. Therefore, in the present study, bone homeostasis was investigated in a hyperinsulinemic, hyperglycemic and obese T2DM mouse model. It was shown in the mouse model that T2DM leads to decreased bone turnover.

The data from the present study show that cortical and trabecular BMD were decreased in the T2DM model. Similarly, Hamann et al (9) previously observed reduced BMD at different skeletal sites in Zucker Diabetic Fatty rats. In addition to the bone analysis, the results from the mechanical testing demonstrated that there was decreased bone strength in mice with T2DM; T2DM mice exhibited reduced ultimate load in the tibia compared with control mice. This suggests that T2DM causes decreased BMD and bone strength, resulting in an increased susceptibility to 
Table II. BMD of cortical bone in C57BL/6 and KK-Ay mice.

\begin{tabular}{lccc}
\hline Parameter & C57BL/6 mice, $n=8$ & KK-Ay mice, $n=8$ & P-value \\
\hline BMD (mg HA/cm) & $1321.7 \pm 108.2$ & $803.4 \pm 87.2^{\mathrm{a}}$ & 0.004 \\
\hline
\end{tabular}

${ }^{\mathrm{a}} \mathrm{P}<0.01$, compared with $\mathrm{C} 57 \mathrm{BL} / 6$ mice. BMD, bone mineral density.

A
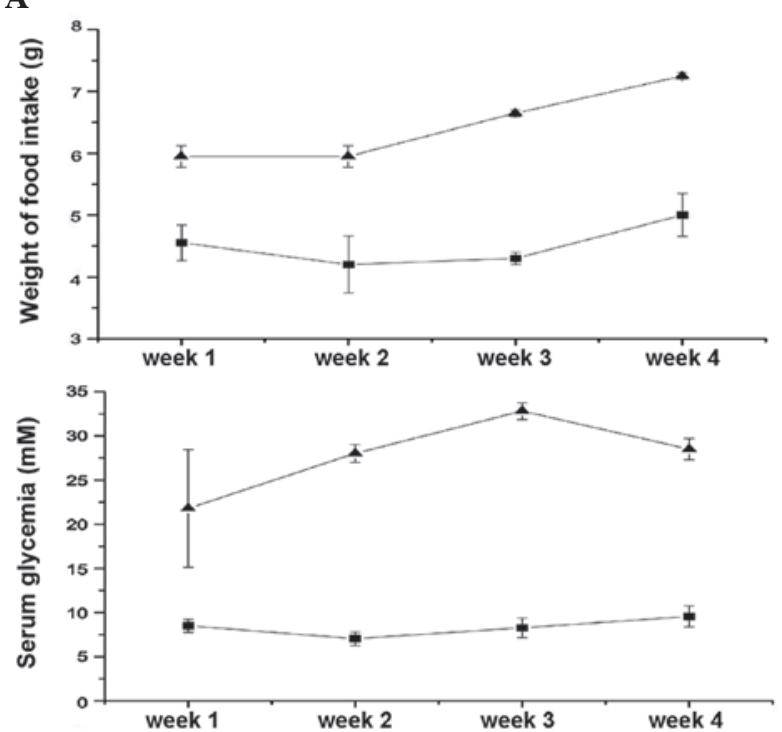

B

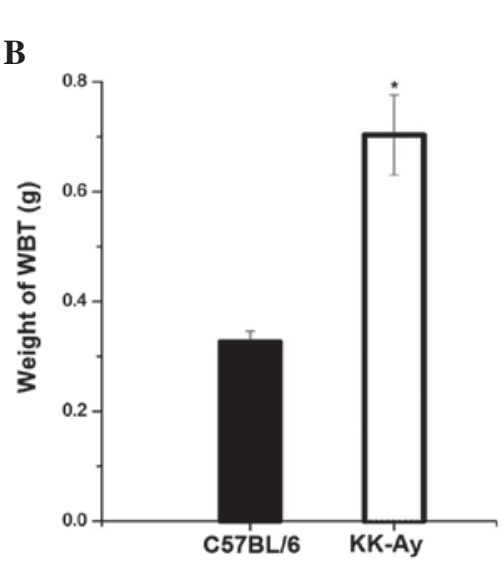

C

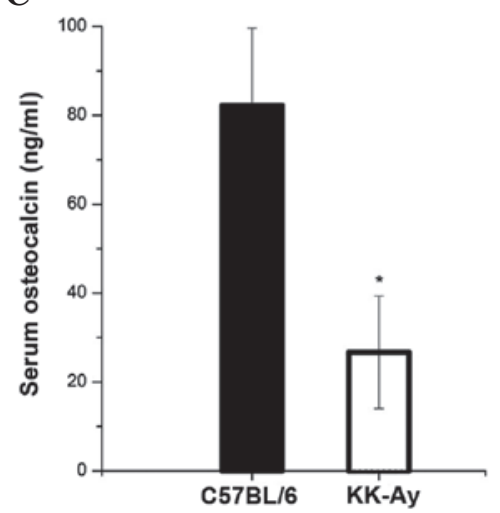

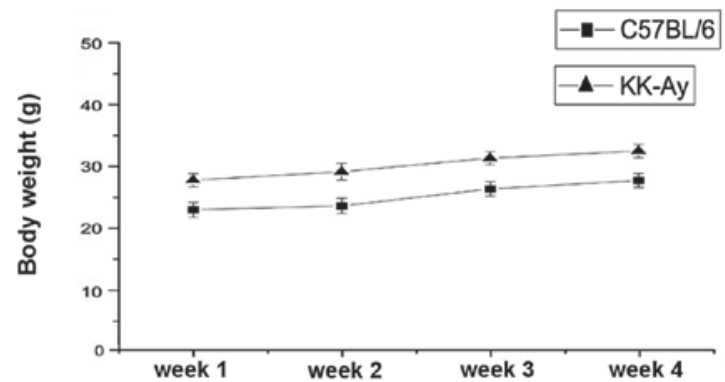

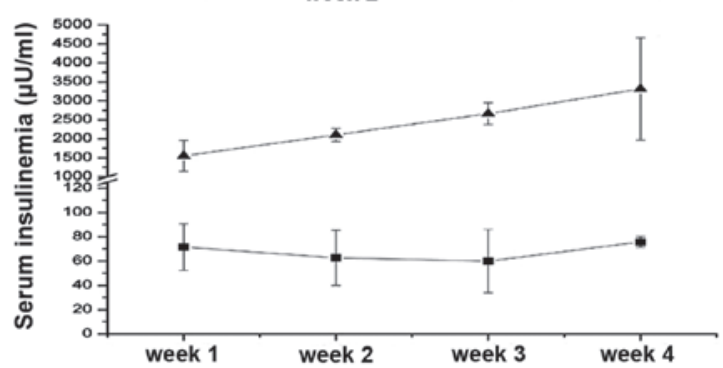

week 1 week 2 week 3 week 4
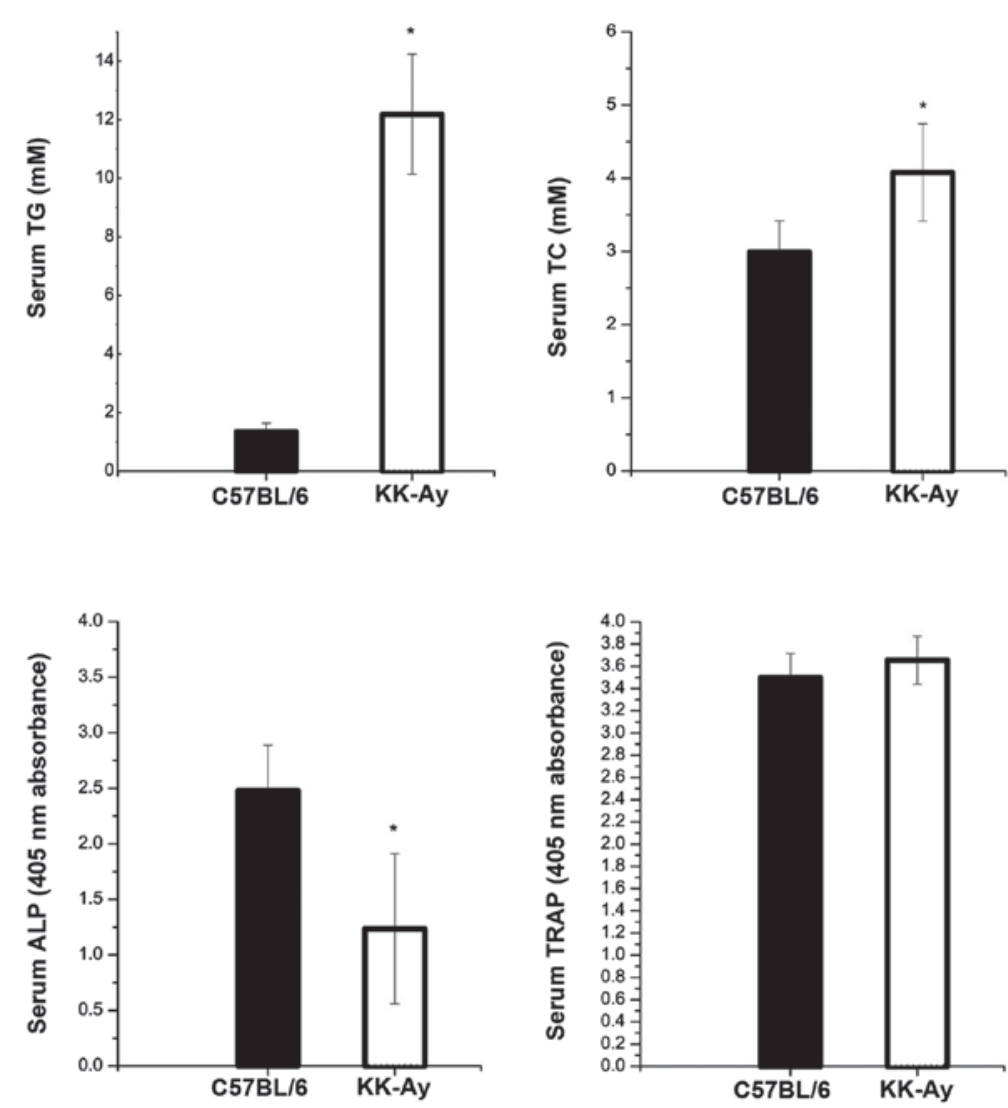

Figure 2. (A) Body weight, food intake and levels of serum glucose and insulin in mice with T2DM and control mice. (B) Topical fat weight and serum TG and TC levels were higher in mice with T2DM compared with those in the control group. (C) Serum osteocalcin, ALP and TRAP levels in mice with T2DM and control mice. " $\mathrm{P}<0.05$, vs. control. T2DM, type 2 diabetes mellitus; ALP, alkaline phosphatase; TRAP, tartrate-resistant acid phosphatase; TG, triglyceride; TC, cholesterol; WBT, weight of the bilateral inguinal fat tissue. 
A

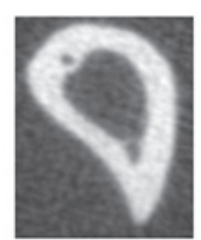

Control

C
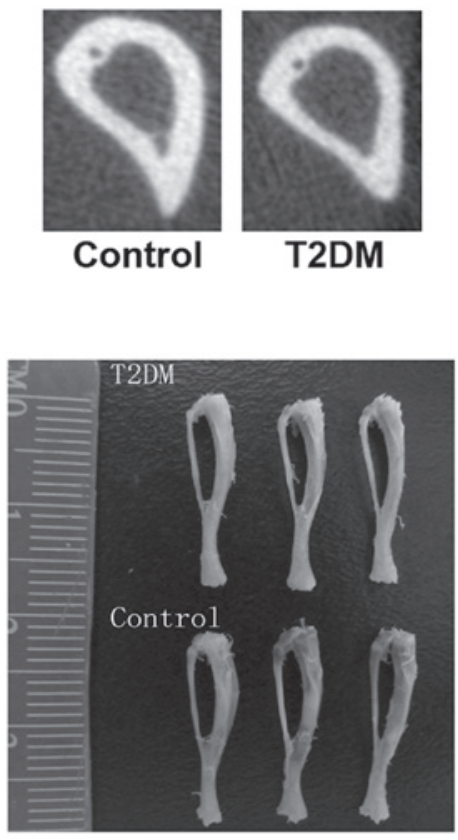

T2DM
B

D

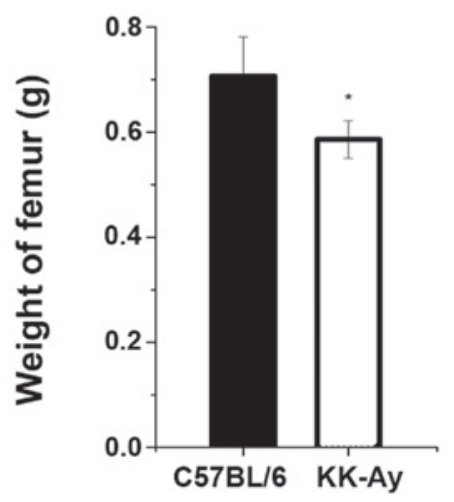

Figure 3. Bone parameters. Section images of (A) the tibia and (B) the L5 vertebral body from mice with T2DM and control mice. (C) Tibiae from mice with T2DM and control mice. (D) The T2DM group had a lower content of bone in the tibia compared with the control group. "P<0.05, vs. control. T2DM, type 2 diabetes mellitus.

A

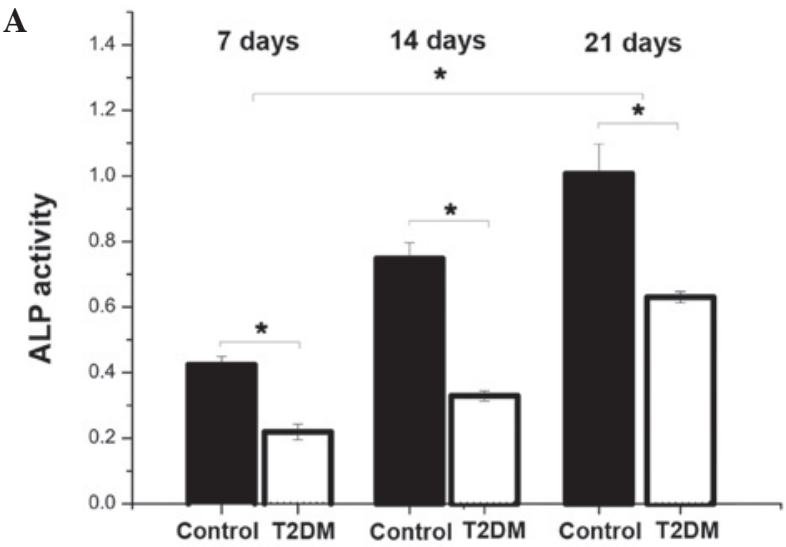

B

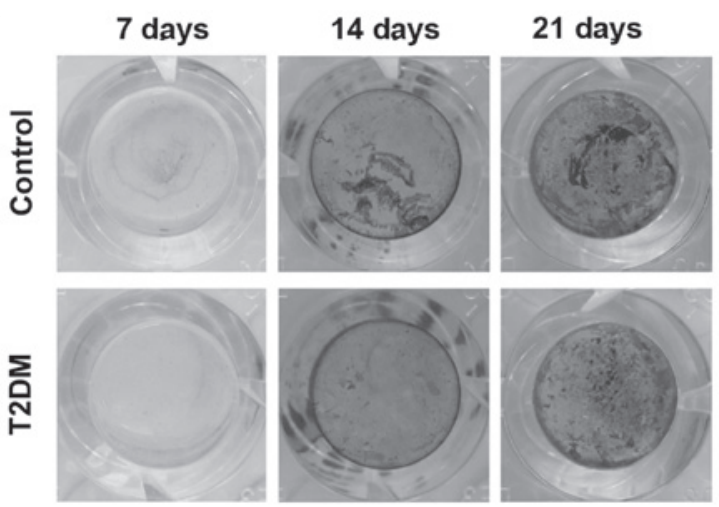

C

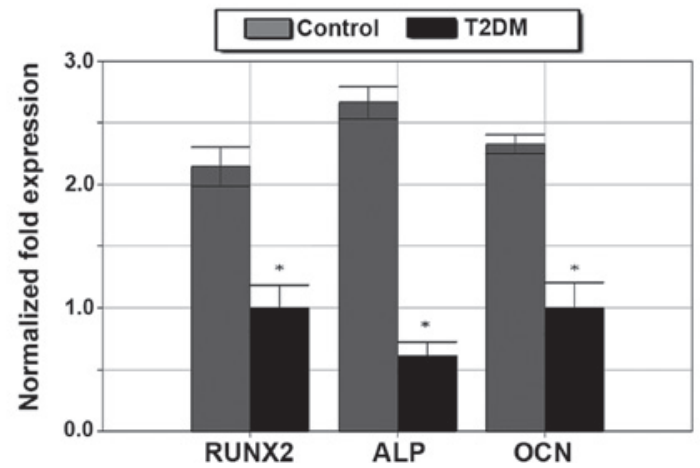

Figure 4. Impaired osteoblast functions in T2DM mice. (A) ALP activity was measured at 7, 14 and 21 days. (B) von Kossa staining of the mineralized matrix was performed after 7, 14 and 21 days. (C) Gene expression of RUNX2, ALP and OCN was normalized against 18S. *P<0.05, vs. control. T2DM, type 2 diabetes mellitus; RUNX2, runt-related transcription factor 2; ALP, alkaline phosphatase; OCN, osteocalcin.

fracture. As a result of T2DM, the impaired bone turnover may be responsible for the high risk of fracture documented in clinical studies $(10,11)$.
The results of the serum analysis in the present study confirm the previously reported impaired bone turnover in T2DM mice $(12,13)$. The present study also showed that the 
A

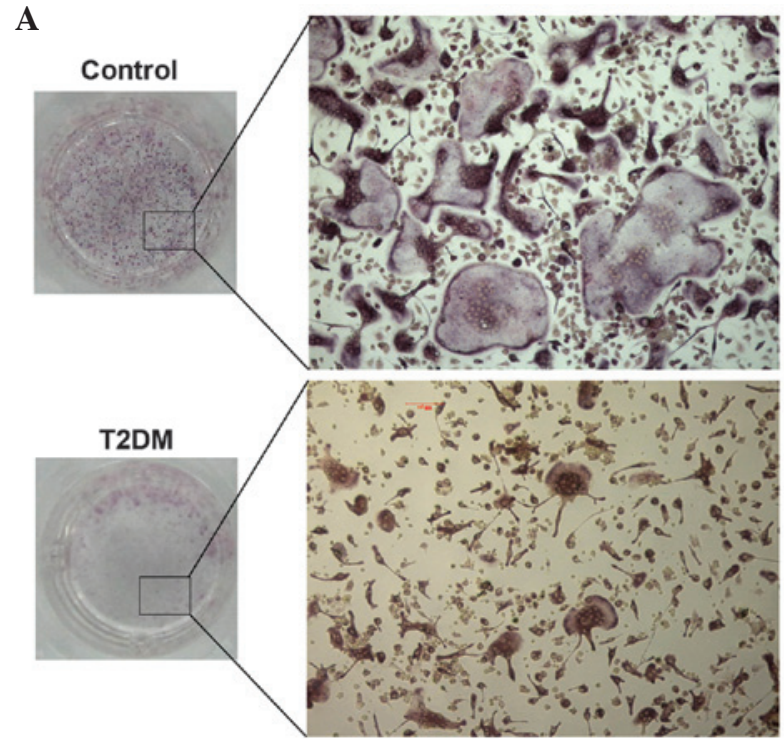

C

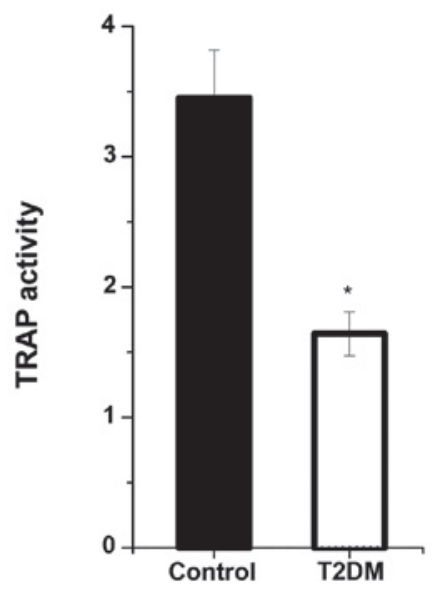

B

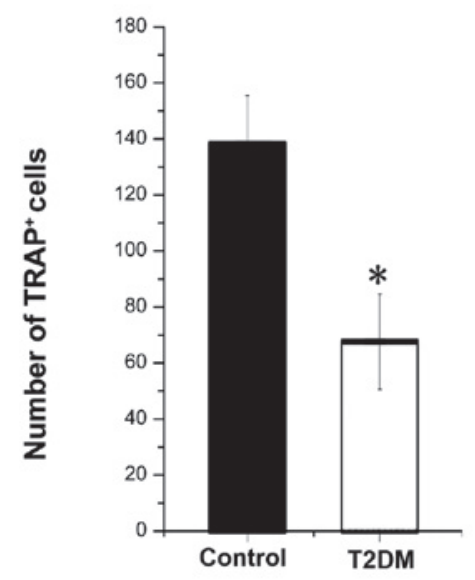

D

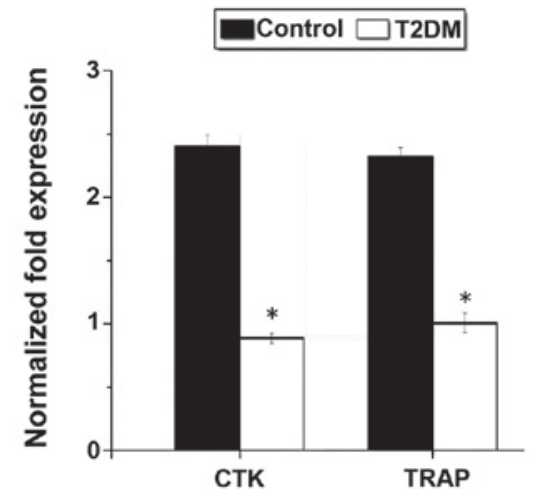

Figure 5. Osteoclast function is attenuated in T2DM mice. (A) Appearance of TRAP ${ }^{+}$osteoclastic cells developed in culture supplemented with M-CSF and RANKL (magnification, x100). (B) Number of TRAP cells developed from nonadherant marrow cells after 4 days of culture in the presence of M-CSF and RANKL. (C) TRAP activity of the osteoclastic cells. (D) Gene expression of CTK and TRAP was normalized to $18 \mathrm{~S}$. "P $<0.05$, vs. control. T2DM, type 2 diabetes mellitus; TRAP, tartrate-resistant acid phosphatase; M-CSF, macrophage colony-stimulating factor; RANKL, receptor activator of nuclear factor $\kappa-B$ ligand; CTK, cathespsin K.

levels of bone turnover and formation, indicated by serum OCN and ALP levels, were lower in T2DM mice than those in normal mice. However, levels of bone resorption, indicated by serum TRAP levels, were not significantly different between the two groups. By contrast, a previous study found that circulating levels of TRAP in patients with diabetes were significantly higher than those in controls (14), while a study investigating the serum bone turnover parameters in patients with T2DM showed reduced bone formation (15) and unchanged bone resorption, assessed by the levels of TRAP. However, evaluating the level of bone metabolism using indicators in serum is not accurate; therefore, in the present study, osteoblastogenesis and osteoclastogenesis were assessed in the two groups of mice.

A previous in vivo study suggested that osteoblast function is impaired in T2DM. Fujii et al (16) reported a lower bone formation rate and decreased gene expression of ALP and OCN in spontaneously diabetic Troii rats, a model of non-obese T2DM (16). The data from the present study provide a cellular explanation for this result: Significant impairment of osteoblastogenesis.
While T2MD is a common disease, there are conflicting studies regarding the influence of T2DM on bone resorption. In vivo, Kawashima et al (17) found evidence supporting increased osteoclastogenesis in the diabetic bone microenvironment (17). In vitro, Huang et al (18) reported that an elevated insulin environment negatively regulated osteoclast differentiation and the expression of RANK and c-fos (18), and these results were corroborated by a study by Dienelt and zur Nieden (19). The data from the present study suggest that bone resorption was impaired in the T2DM model due to impaired osteoclast function. In addition, using monocytes derived from normal animals, osteoclastogenesis was assessed under high-glucose and insulin environments. The expression of osteoclast gene markers, including CTK, TRAP and RANK, was found to be decreased (data not shown). Along with the results from the TRAP staining, these findings indicate that osteoclastogenesis in mice with T2DM was decreased.

During the early stages of T2DM, the majority of patients are overweight. Fat tissue is targeted by insulin and has a key role in insulin resistance. Adipocytes also secrete factors that inhibit the differentiation of osteoblasts (20), and a high-insulin 
environment is a negative factor for bone formation (18) Therefore, for these reasons, obesity is a promoting factor for osteoporosis in T2DM.

The present study had certain limitations. Firstly, the extent of impaired of bone resorption and formation could not be evaluated. In addition, young mice were used for these experiments, and the study only lasted for 4 weeks. The results may not be consistent in older mice, and most patients with T2DM in the clinic are aged $\geq 30$ years. Finally, the effect of the microenvironment of the surrounding bone on the cells was not investigated. Future studies may therefore explore osteoclastogenesis and osteoblastogenesis in co-culture systems. However, the results offer a novel insight into the cellular mechanisms underlying T2DM pathology. The results suggest that a T2DM mouse model exhibits bone loss predominantly due to suppressed bone formation, rather than increased resorption. The hyperglycemia and hyperinsulinemia in KK-Ay mice models the human pathology, enhancing the current understanding of impaired bone metabolism in T2DM.

\section{Acknowledgements}

This study was supported by a grant from the National Natural Science Foundation of China (no. 81070691).

\section{References}

1. Yamaguchi $\mathrm{T}$ and Sugimoto $\mathrm{T}$ : Bone metabolism and fracture risk in type 2 diabetes mellitus. Endocr J 58: 613-624, 2011.

2. Forsén L, Meyer HE, Midthjell K and Edna TH: Diabetes mellitus and the incidence of hip fracture: results from the Nord-Trøndelag Health Survey. Diabetologia 42: 920-925, 1999.

3. Retzepi M and Donos N: The effect of diabetes mellitus on osseous healing. Clin Oral Implants Res 21: 673-681, 2010.

4. Lecka-Czernik B: Bone loss in diabetes: use of antidiabetic thiazolidinediones and secondary osteoporosis. Curr Osteoporos Rep 8: 178-184, 2010

5. Horcajada-Molteni MN, Chanteranne B, Lebecque $P$, Davicco MJ, Coxam V, Young A and Barlet JP: Amylin and bone metabolism in streptozotocin-induced diabetic rats. J Bone Miner Res 16: 958-965, 2001.

6. Suzuki K, Sugimoto C, Takizawa M, Ishizuka S, Kikuyama M, Togawa H, Taguchi Y, Nosaka K, Seino Y and Ishida H: Correlations between bone mineral density and circulating bone metabolic markers in diabetic patients. Diabetes Res Clin Pract 48: 185-191, 2000.

7. Wakasugi M, Wakao R, Tawata M, Gan N, Koizumi K and Onaya T: Bone mineral density measured by dual energy $\mathrm{x}$-ray absorptiometry in patients with non-insulin-dependent diabetes mellitus. Bone 14: 29-33, 1993.
8. Barrett-Connor E and Holbrook TL: Sex differences in osteoporosis in older adults with non-insulin-dependent diabetes mellitus. JAMA 268: 3333-3337, 1992.

9. Hamann C, Goettsch C, Mettelsiefen J, Henkenjohann V, Rauner M, Hempel U, Bernhardt R, Fratzl-Zelman N, Roschger P, Rammelt S, Günther KP and Hofbauer LC: Delayed bone regeneration and low bone mass in a rat model of insulin-resistant type 2 diabetes mellitus is due to impaired osteoblast function. Am J Physiol Endocrinol Metab 301: E1220-E1228, 2011.

10. Strotmeyer ES, Cauley JA, Schwartz AV, Nevitt MC, Resnick HE, Bauer DC, Tylavsky FA, et al: Nontraumatic fracture risk with diabetes mellitus and impaired fasting glucose in older white and black adults: the health, aging, and body composition study. Arch Intern Med 165: 1612-1617, 2005.

11. Bonds DE, Larson JC, Schwartz AV, Strotmeyer ES, Robbins J, Rodriguez BL, Johnson KC and Margolis KL: Risk of fracture in women with type 2 diabetes: the Women's Health Initiative Observational Study. J Clin Endocrinol Metab 91: 3404-3410, 2006.

12. Zhou Y, Li Y, Zhang D, Wang J and Yang H: Prevalence and predictors of osteopenia and osteoporosis in postmenopausal Chinese women with type 2 diabetes. Diabetes Res Clin Pract 90: 261-269, 2010.

13. Kanazawa I, Yamaguchi T, Yamamoto M, Yamauchi M, Yano S and Sugimoto T: Serum osteocalcin/bone-specific alkaline phosphatase ratio is a predictor for the presence of vertebral fractures in men with type 2 diabetes. Calcif Tissue Int 85: 228-234, 2009.

14. Takizawa M, Suzuki K, Matsubayashi T, Kikuyama M, Suzuki H, Takahashi K, Katsuta H, Mitsuhashi J, Nishida S, Yamaguchi S, Yoshimoto K, Itagaki E and Ishida H: Increased bone resorption may play a crucial role in the occurrence of osteopenia in patients with type 2 diabetes: Possible involvement of accelerated polyol pathway in its pathogenesis. Diabetes Res Clin Pract 82: 119-126, 2008.

15. Okazaki R, Miura M, Toriumi M, Taguchi M, Hirota Y, Fukumoto S, Fujita T, Tanaka K and Takeuchi A: Short-term treatment with troglitazone decreases bone turnover in patients with type 2 diabetes mellitus. Endocr J 46: 795-801, 1999.

16. Fujii H, Hamada Y and Fukagawa M: Bone formation in spontaneously diabetic Torii-newly established model of non-obese type 2 diabetes rats. Bone 42: 372-379, 2008.

17. Kawashima Y, Chen J, Sun H, Lann D, Hajjar RJ, Yakar S and Leroith D: Apolipoprotein E deficiency abrogates insulin resistance in a mouse model of type 2 diabetes mellitus. Diabetologia 52: 1434-1441, 2009.

18. Huang S, Kaw M, Harris MT, Ebraheim N, McInerney MF, Najjar SM and Lecka-Czernik B: Decreased osteoclastogenesis and high bone mass in mice with impaired insulin clearance due to liver-specific inactivation to CEACAM1. Bone 46: 1138-1145, 2010.

19. Dienelt A and zur Nieden NI: Hyperglycemia impairs skeletogenesis from embryonic stem cells by affecting osteoblast and osteoclast differentiation. Stem Cells Dev 20: 465-474, 2011.

20. Elbaz A, Wu X, Rivas D, Gimble JM and Duque G: Inhibition of fatty acid biosynthesis prevents adipocyte lipotoxicity on human osteoblasts in vitro. J Cell Mol Med 14: 982-991, 2010. 